\title{
Dense Small-Cell Networks: Rethinking the Radio Interface Beyond LTE-Advanced
}

\author{
Toni Levanen*, Juho Pirskanen ${ }^{\dagger}$, and Mikko Valkama* \\ *Tampere University of Technology, Tampere, Finland \\ ${ }^{\dagger}$ Broadcom Corporated, Finland \\ e-mail: toni.levanen@tut.fi
}

\begin{abstract}
In this article, we provide an overview of the 5GETLA radio interface proposal for low latency dense smallcell networks. We discuss the new physical layer parametrization and frame design to support high energy and spectral efficiency in wireless small-cell communications and compare the main parameters with another recent physical layer design proposed for $5 \mathrm{G}$ centimeter wave communications. The challenges and potential of the design proposals are opened and discussed. The main emphasis in these designs is to go beyond LTE-Advanced to achieve 5G design targets, while leaving room for further innovations.
\end{abstract}

Index Terms-5G, dense networks, energy efficiency, flexible TDD, local area, LTE/LTE-Advanced, low latency, small cell networks, spectral efficiency

\section{INTRODUCTION}

Wireless communications system design has three main dimensions, namely spectral efficiency (SE), energy efficiency (EE), and latency or the round trip time (RTT) of physical layer (PHY) packets. As indicated in [1], and in several other surveys, the expected data traffic growth during the next decade will most likely be exponential. This kind of growth, while maintaining profitable operation for network vendors, is not possible unless there are new system designs providing significant improvements on the SE and EE. In general, going towards substantially increased density of small local area (LA) cells or small-cell networks ( $\mathrm{SCN}$ ) is seen as one key trend to solve the exponentially growing data challenge.

In addition to SE and EE targets, we also have to minimize the PHY latency of the new wireless systems to achieve RTT smaller than $1 \mathrm{~ms}$, as indicated in [1], [2]. There are two main drivers for the very low latency of the new radio interface. Firstly, new applications enabled by 5G wireless networks providing augmented reality, new level of mobile gaming, cloud services and much more demand for reduced latency. Secondly, the manufacturing industry in general is starting to realize the possible benefits of cheap and fast wireless access for surveillance and control, and impose strict reliability and latency requirements for the new wireless network design [3].

In this article, we provide an overview of a new radio interface introduced in [4], based on orthogonal frequency division multiple access (OFDMA) and flexible time division duplexing (TDD), entitled 5G flexible TDD based local area (5GETLA). Also, we compare the 5GETLA physical layer parametrization and frame design with a recent radio interface proposal by Mogensen et al. [5]-[8], which will be referred as the B4G design in this paper. In both designs the downlink

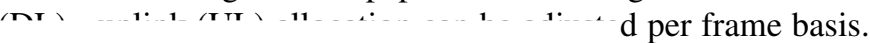

In addition, both designs use well known tools to provide new numerologies that push the limits of OFDMA based scheduled and synchronized wireless access to boost the EE and SE when compared to LTE [9], [10] and WLAN 802.11ac [11], [12]. Also, they provide PHY latencies in the order of $1 \mathrm{~ms}$, leading to an order of magnitude improvement in the PHY latency when compared to LTE. Already in [1], it was indicated that there is a need for solutions clearly reducing the symbol and frame duration when compared to LTE. Both designs are also evaluated from the dense wireless networks perspective and we discuss the nature of traffic and interference in this environment and explain why we believe that flexible TDD is best suited for this task. More general discussion on the open research topics related to $5 \mathrm{G}$ radio interface can be found, e.g., from [13], in which the research directions of the Mobile and wireless communications Enablers for the Twenty-twenty Information Society (METIS) project are elaborated.

This article is organized as follows. In Section II, we start by introducing design challenges related to dense SCN. In Section III, we introduce the new radio interfaces and compare their frame design and parametrization. Then, in Section IV we open up the possibilities the new designs provide and describe how they map to SE, EE, and latency improvements. Finally, in Section $\mathrm{V}$ we wrap up the main benefits of the new radio interfaces and indicate the main open research topics related to the PHY design for future dense SCN.

\section{Design Challenges for Dense Small-Cell NETWORKS}

\section{A. Inter-Cell Interference in Ultra-Dense Networks}

Going towards extremely dense SCN is inevitably going to signify the effect of interference on the system level performance. Assuming that we have significantly overlapping 5G small cells, each dynamically adjusting their DL-UL allocation per frame basis without any cooperation, the question is that how much traffic does the system tolerate before the interference starts to saturate the achievable throughput and what is the average cell capacity with different amounts of accumulated traffic.

In Fig. 1, the three different types of interference encountered in dynamic TDD networks are shown. The DL-DL and UL-UL interference is common for any wireless network and are always present in wireless communications. In flexible TDD, as in any uncoordinated TDD based network, the very high dynamics of the UL-DL interference type is an unsolved problem. On the other hand, in a flexible TDD scenario with 


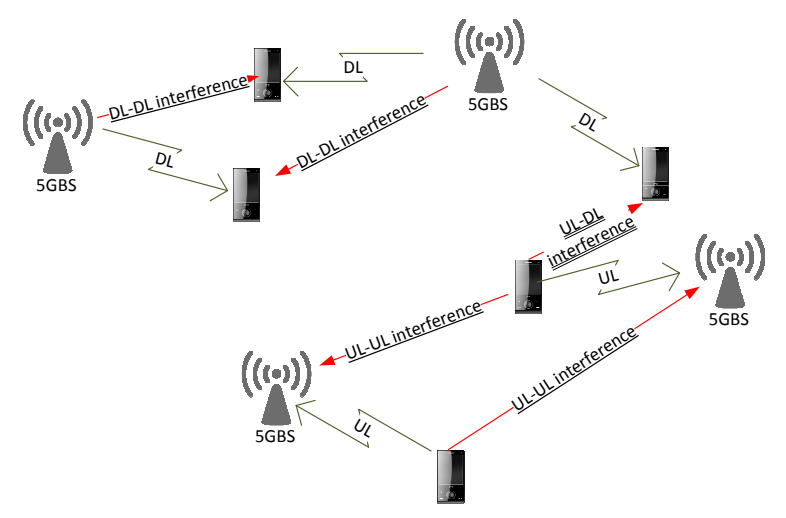

Fig. 1. Different interference types encountered in flexible TDD based dense networks.

a very fast PHY we can assume that only a fraction of the received packets are destroyed by the alignment of our DL reception and the UL transmission of a nearby UE. If a UE nearby is transmitting to its own $5 \mathrm{G}$ base station (5GBS) with high power, the received UL-DL interference power may exceed the dynamic range of the receiver and completely block the reception. If the dynamic range of the receiver is sufficient, then it depends on the interference cancellation (IC) algorithm how well the weaker signal can be detected after removing the significantly stronger interferer. Typically, IC algorithms work well in the presence of strong power differences in the received signals so the main focus is in the accuracy of the receiver frontend and if it is able to provide sufficient information of the low power desired signal [6]. A more common higher level solution is a network controller that takes care that UL-DL interference does not occur in the network. Also, distributed coordination for the DL-UL allocation decisions (see for example [14]) can be considered. The question to answer is how much realized improvement we obtain in the system throughput if we use either distributed or centralized coordination when compared to the uncoordinated case with or without IC capabilities.

We believe that providing a fast TDD based radio interface with a per-frame DL-UL allocation reconfiguration capability enables the 5GBS to quickly serve UE as they receive packet to send in DL or UL. Under the assumption of unsynchronized packet arrival processes in the UE, the question is how much traffic can be generated in the network before the Tx buffers start to grow leading to full buffer like environment. It is obvious, that for full buffer traffic a fixed DL-UL allocation based on the average DL/UL traffic ratio of the network provides the best results. In addition, understanding the signalto-interference-and-noise (SINR) statistics in very dense SCN with radio interfaces capable of reconfiguring the DL-UL allocation per frame, frame durations being $0.5 \mathrm{~ms}$ or less, is an open issue that needs to be carefully studied.

\section{B. Sporadic Small Packet Traffic}

As the number of devices served increases and the number of applications that require constant status updates in these devices increases, the amount of sporadic small packet traffic exponentially grows in the future wireless networks. Also sensor networks and different industry applications request efficient implementation for asynchronous low rate data streams. Preparing for this already in the system design phase is crucial. In the 5GETLA design defined in Section III, a combined random access channel (RACH) and contention based data channel $(\mathrm{CBDCH})$ is proposed in which the devices may send best effort contention based small data packets without requesting for resource allocation. To support large number of $\mathrm{CBDCH}$ packets, we can increase the $\mathrm{RACH} / \mathrm{CBDCH}$ allocation and reduce the capacity of the scheduled access. Using a robust modulation and coding scheme (MCS) for the $\mathrm{CBDCH}$ packets allows the possibility of detecting multiple simultaneous transmissions using similar algorithms that would be used for IC. In the 5GETLA design the overhead of $\mathrm{RACH}$ messages and $\mathrm{CBDCH}$ packets is minimized by allocating them to the same time-frequency resources because they have time diversity from the asynchronous generation process and are easier to detect based on known structure of $\mathrm{RACH}$ sequences and robust MCS of the $\mathrm{CBDCH}$ packets. Also other possibilities for supporting the sporadic traffic exists. For example, in [15], new waveforms were presented to support low rate asynchronous traffic with minimal timefrequency allocation but they are not considered here due to the increased signal processing burden in the receiver.

\section{Hardware Limitations}

To fully benefit from the very fast frame structure designed for the $5 \mathrm{G}$ radio interface, the hardware designed for future mobile devices should be fast enough to claim the gains offered by the design. For example, it depends on different components how efficiently sleep times can be utilized and how much time overhead is required for, e.g., power amplifier ramp times [8], or oscillator settling times and synchronization times after device wakes up from different sleep modes. Mapping the desired performance to hardware specifications is an open research task which is required to provide us a roadmap defining how much and which components should be improved to fully gain from very fast, low latency system designs. Furthermore, to fully capitalize the gains of the agile radio interface we may have to reconsider the scheduling algorithms used in the forthcoming $5 \mathrm{G} \mathrm{SCN}$.

In addition, the current design of 5GETLA frame structure sets strict timing requirements for detecting physical layer packets and generating acknowledgment (ACK) or negative ACK indicators in the 5GBS with delays in the order of tens of microseconds. The computational requirements become even stricter with multiple-input multiple-output (MIMO) transmissions. To alleviate the processing requirements in low class devices and to have more time for decoding and detecting transmitted packets, we can schedule empty intervals into the center part of the 5GETLA frame or increase the $\mathrm{RACH} / \mathrm{CBDCH}$ allocation. This is then observed as reduced $\mathrm{SE}$ for the low class devices. In contrast to the frame design presented in [16], the frame design in Fig. 2 places the uplink control channel (ULCCH) after the RACH/CBDCH, which provides UE automatically with more time to finish 

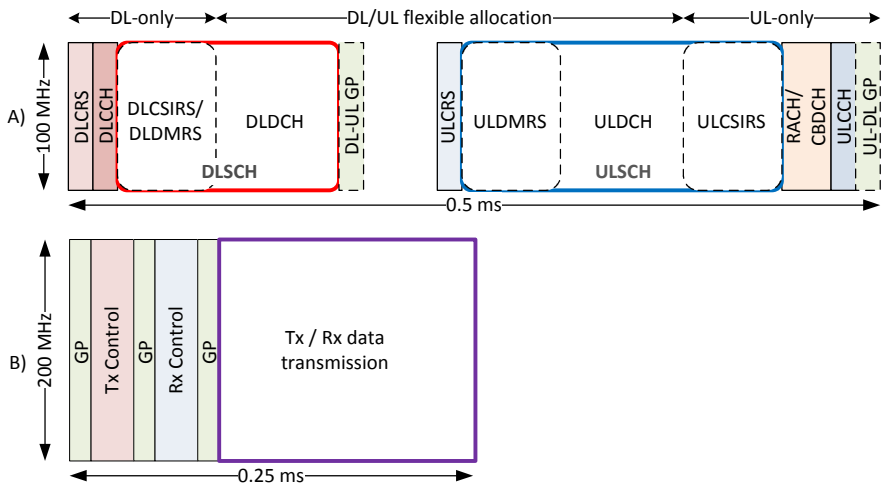

Fig. 2. Frame design for the A) 5GETLA and B) B4G.

decoding of DL packets before generating DL ACK indicators. In general, the 5GETLA radio interface should be easily implementable because the OFDM numerology used is very similar to the $802.11 \mathrm{ac}$ with $160 \mathrm{MHz}$ channel bandwidth. In the B4G design the receiver always has one frame duration of processing time before generating an ACK, thus providing a more relaxed timing requirements, as shown in Fig. 3.

\section{Spectrum Allocation}

If we want to discard the backward compatibility and provide full throughput gain promised by the new radio interface designs, we have to find a new spectrum allocation for any new $5 \mathrm{G}$ system. Currently the spectrum below $3 \mathrm{GHz}$ is congested and it is hard to find contiguous allocations for systems with at least $100 \mathrm{MHz}$ of system bandwidth. At $3 \mathrm{GHz}$ and $5 \mathrm{GHz}$ there are possible frequency bands in which the 5GETLA system could be allocated. For example, in the EUTRA operating bands $42(3400 \mathrm{MHz}-3600 \mathrm{MHz})$ and $43(3600 \mathrm{MHz}-3800 \mathrm{MHz})$ [17, Table 5.5-1], which are defined for TDD duplex based mode for LTE-A could provide $400 \mathrm{MHz}$ of contiguous spectrum for $5 \mathrm{G}$ communications.

The $5 \mathrm{G}$ radio interfaces presented in this paper are designed to operate under $30 \mathrm{GHz}$ carrier frequency. Especially, the 5GETLA is designed by fitting the physical layer numerology to channel models measured below $10 \mathrm{GHz}$. Recently, the vast spectrum available above $30 \mathrm{GHz}$ carrier has become a subject for intensive research. The millimeter wave ( $\mathrm{mm}$-wave) mobile broadband systems [18] provide tens of gigahertz of vacant spectrum. There are still several implementation and design challenges, but the initial results seem promising and the near future may witness the birth of $\mathrm{mm}$-wave communications for mobile devices. The 5GETLA frame design is on high level very similar to the one proposed in [18], and with fine tuning on the physical layer numerology we can adapt it also for mm-wave broadband systems (e.g. [19]).

\section{New Radio Interfaces for 5G SMall-Cell NETWORKS}

The main ideas behind 5G physical layer designs presented here are to have scheduled, synchronized, and flexible TDD based OFDMA radio system that is fast and spectrally efficient. The assumption of the scheduled and synchronized
TABLE I

MAIN PARAMETERS FOR 5GETLA AND B4G RADIO INTERFACES. THE VALUES USED FOR B4G ARE OBTAINED FROM [6, TABLE 1.] OR DERIVED FROM THE VALUES PRESENTED THERE.

\begin{tabular}{|l|cc|}
\hline \multirow{2}{*}{ Parameter } & \multicolumn{2}{|c|}{ Value } \\
& 5GETLA & B4G \\
\hline$F_{s}:$ Sampling frequency [MHz] & 92.16 & 245.76 \\
$N_{F F T}:$ FFT size & 512 & 4096 \\
$T_{F F T}:$ FFT duration [ $\mu$ s] & 5.56 & 16.67 \\
$N_{C P}:$ Cyclic prefix length (long/short) & $32 / 30$ & 246 \\
$T_{C P}:$ Cyclic prefix duration (long/short) [ns] & $347 / 326$ & 1001 \\
$N_{G P}:$ Guard period length (long/short) & $544 / 542$ & $218 / 217$ \\
$T_{G P}:$ Guard period duration (long/short) [ns] & $5903 / 5882$ & $887 / 883$ \\
$\Delta_{\text {sc }}:$ subcarrier spacing [kHz] & 180 & 60 \\
$B_{s}:$ System bandwidth [MHz] & 100 & 200 \\
$\alpha_{\text {new }}:$ Bandwidth utilization factor & 0.9 & 0.99 \\
$T_{\text {slot }}:$ Slot duration [ms] & 0.1 & - \\
$N_{S p S}:$ Symbols per slot & 17 & - \\
$T_{\text {frame }}:$ Frame duration [ms] & 0.5 & 0.25 \\
$N_{S p F}:$ Symbols per frame & 85 & 14 \\
GP+CP overhead [\%] & 7.9 & 6.7 \\
$T_{\text {long } \text { frame: Long frame duration [ms] }}:$ & 10 & - \\
$T_{\text {master frame }: \text { Master frame duration [ms] }}$ & 100 & - \\
$N_{R B}:$ Number of resource blocks & 5 & - \\
$\Delta f_{R B}:$ Frequency allocation per RB [MHz] & 18 & - \\
\hline
\end{tabular}

frame based wireless access comes from the 3GPP LTE design, which has proven to be an efficient scheme to serve large populations of UE. Flexible TDD with per frame reconfiguration is seen as a potential candidate for SCN because, as shown in several sources (e.g. [20]), the faster the DL-UL allocation reconfiguration time is the better is the throughput performance with finite buffer traffic for networks based on LTE TDD frame structure. This is because the small-cell traffic dynamics change dramatically as the associated UEs download or upload content at different time instances. Providing a system design in which we can change the DL-UL allocation per $0.5 \mathrm{~ms}$ or $0.25 \mathrm{~ms}$ frame duration (see Table I), the system can efficiently adapt to instantaneous traffic orientation to faster satisfy data transfer requirements and maximize sleeping times for network and UE side.

\section{A. Physical Layer Numerology}

The 5GETLA physical layer design is based on a large survey on the LA environment characteristics and channel models, and the assumption that the UEs connected to the system have low mobility (less than $30 \mathrm{~km} / \mathrm{h}$ ) [4]. The low mobility assumption is clearly more restrictive than in current LTE, but the open question is that can we support high mobility users through massive beamforming (narrow beams) and if so, how much denser reference symbol (RS) layout is required. The main physical layer parameters for both designs are given in Table I. The sampling frequencies are integer multiples of the LTE sampling frequency to be easily obtained from the LTE system clock. We consider the 5GETLA design as a revolutionary step based on the current 3GPP LTE evolution, with some design aspects assimilated from WLAN 802.11 physical layer numerology [4]. The FFT size $N_{F F T}$, is kept as small as possible to provide significantly shorter OFDM 


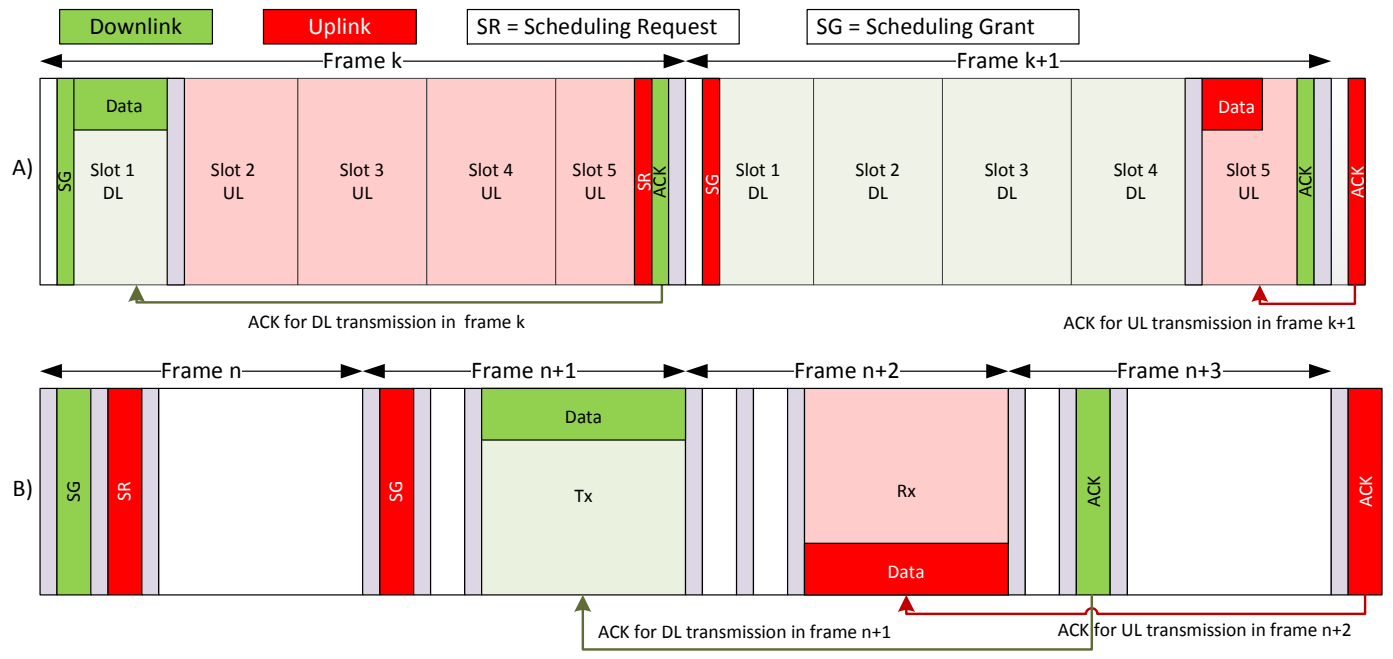

Fig. 3. DL and UL ACK timing examples for the A) 5GETLA and B) B4G frame designs.

symbol durations than in LTE, while maintaining cyclic prefix (CP) overhead at approximately $5 \%$. In B4G, assumption of $200 \mathrm{MHz}$ contiguous system bandwidth is made. In addition, the OFDM and CP durations are clearly reduced from LTE numerology although being longer than in 5GETLA, allowing larger cells and better tolerance towards intercell interference. Currently, in the 5GETLA design the GP is one OFDM symbol long causing extensive overheads and room for improvement.

Due to relatively short channel delay spreads encountered in LA environment, the frequency correlation is significant over several $\mathrm{MHz}$ and gains achieved by frequency scheduling are smaller than in macro cells. Therefore, and to limit the scheduling overhead, the active band of $90 \mathrm{MHz}$ is divided into $N_{R B}=5$ resource blocks (RB) in 5GETLA. Further details on the average SNR gains with different RB and total allocation sizes are given in [4]. Typically, we lose in average SNR gain with larger RB, whereas we save in the scheduling and feedback overhead because the feedback is done per RB basis. In 5GETLA system design, RB defines the frequency allocation but time allocation is separately indicated by the DL-UL allocation factor. Thus, even having the same RB and MCS in successive frames the actual throughput per frame per UE may change based on the frame-wise DL-UL allocation. The DL-UL allocation accuracy can be per slot or per OFDM symbol. By using per OFDM symbol allocation we slightly increase the control overhead while improving the utilization of the time resources. Another approach would be to fix the transmission direction per frame to be either DL or UL, thus fixing the interference statistics over the frame. This approach has been used in the B4G design [5]-[7], as indicated in Fig. 2. This setup is also possible as a special case of the 5GETLA design. The comparison of achievable throughput or cell capacity with either fixed or variable DL-UL allocation per frame is important although open research topic in ultradense SCN.

\section{B. Frame Design}

The 5GETLA frame duration is set to $0.5 \mathrm{~ms}$ and $\mathrm{B} 4 \mathrm{G}$ frame duration is $0.25 \mathrm{~ms}$, to achieve the $1 \mathrm{~ms}$ latency target set for
5G communications at the physical layer. The 5GETLA and B4G frame designs are illustrated in Fig. 2.

In 5GETLA design [4], the frame starts with the DL (Tx) section and is followed by the UL (Rx) section. The DL-UL allocation is adjusted per frame basis. In DL, downlink common reference symbol (DLCRS) and downlink control channel (DLCCH) are compulsory fields to be broadcasted when the 5GBS is active. The downlink shared channel (DLSCH) contains the downlink data channel (DLDCH) and in the case of MIMO transmission it may contain also downlink channel state information reference symbols (DLCSIRS) or downlink demodulation reference symbols (DLDMRS). Further details on RS allocation in the case of MIMO transmission is provided in [16]. The cell-wise DL-only part of the frame is defined by the number of DLCRS, DLCCH, and DLCSIRS or DLDMRS symbols. In the case of cooperation among 5GBS, they can agree on common values for DL-only and UL-only time windows in a small neighborhood to improve the detection reliability on these symbols. The main purpose of these time windows is to enable transmissions protected from DL-UL interference. The DL section ends with the DL-UL GP and may be followed by an empty time interval, during which the is no active Tx or Rx in the scheduled links.

The UL section starts with the uplink common reference symbols (ULCRS), which is followed by the uplink shared channel (ULSCH). ULSCH contains the uplink data channel (ULDCH) and in the case of MIMO transmission [16] also the uplink channel state information reference symbols (ULCSIRS) or uplink demodulation reference symbols (ULDMRS). The ULDMRS is now located in the beginning of the UL section to alleviate the design of a more efficient pipelined hardware implementation. The RACH channel is used for association or for UL scheduling requests (SR). The contention based data channel (CBDCH), as discussed in [4], is a novel contention based channel providing a tool to support ultralow latency, best-effort channel for small packet transmissions. The sporadic small packet traffic is often overlooked in the traffic models and system designs for next generation wireless access. For example, in [21] it was shown that 35\%-95\% 
of the application specific UL data bursts were less or equal to 100 bytes. Also, in our own measurements we have noted a large number of small packets being transmitted and received when downloading a Youtube video stream. In addition, the allocation for the $\mathrm{CBDCH}$ can be made to cover most of the available resources in a 5GETLA frame and as a special case the UL traffic in a cell may be only $\mathrm{CBDCH}$ packets, which provides interesting possibilities especially for sensor and industry applications. The 5GETLA frame ends with the uplink control channel (ULCCH) and UL-DL GP.

In the B4G frame design [5]-[8], the high level description is very simple. The frame starts with a GP followed by a Tx Control field. Then GP and Rx Control field, and finally GP and the data field supporting either Tx or Rx data packets. Based on the authors knowledge, no finer details on the control fields have been published. In the case that the 5GBS is the transmitting entity in the Tx Control and UE is the transmitting entity in the Rx Control, these control fields correspond to the traditional DL and UL control fields, respectively. In B4G, the transmission link can also exist between two 5GBS or two $\mathrm{UE}$, and then the connection corresponds to self-backhauling or device-to-device communications, respectively [5]. Also, in 5GETLA design these communication modes are possible assuming that one device acts as a connection master being responsible for the scheduling.

In Fig. 3, we show the assumed delays and timing constraints for DL and UL transmission in both designs. In the case of 5GETLA (part A), the transmission of the DL data follows immediately after the DL scheduling grant (SG) provided in the DLCCH. This minimizes the detection latency, but the downside is that the UE has to buffer the Rx signal until it has detected the DLCCH message and confirmed if there is a scheduled transmission for it. The ACK for the DL data transmission is transmitted in the same frame inside the ULCCH message. This leads to PHY RTT of approximately $0.5 \mathrm{~ms}$. For the UL, the SR is indicated through the RACH after which a SG is provided in the beginning of the next frame to minimize the PHY latency of the first packets. Because the scheduler does not know the buffer status of the UE requesting resources, the initial UL allocation size depends on the status of the other served UE. For example, in part A of Fig. 3, the UL SR is transmitted in the end of frame $k$, the UL SG and data transmission takes place in the frame $k+1$, and the ACK for the UL data transmission is provided in the DLCCH of the frame $k+2$ leading to a PHY RTT in the order of $0.5 \mathrm{~ms}$.

In the case of the B4G design, as indicated in Fig. 3 B), the DL SG is provided in frame $n$, the DL data is transmitted in frame $n+1$, and the related ACK is transmitted in the $\mathrm{Rx}$ control field of the frame $n+3$ leading to approximately $0.75 \mathrm{~ms}$ PHY RTT. In the case of UL transmission, the UL $\mathrm{SR}$ is transmitted in frame $n$, UL SG in frame $n+1$ which schedules the UL data transmission to frame $n+2$, and the related ACK is transmitted in the frame $n+4$, achieving RTT of approximately $1 \mathrm{~ms}$. The good aspect of the B4G design is that because the SG always points to the next frame, the UE has to listen only to the Tx Control and can then turn off the $\mathrm{Rx}$ chain. The downside is that this type of scheduling inherently increases the PHY latency when evaluated as the

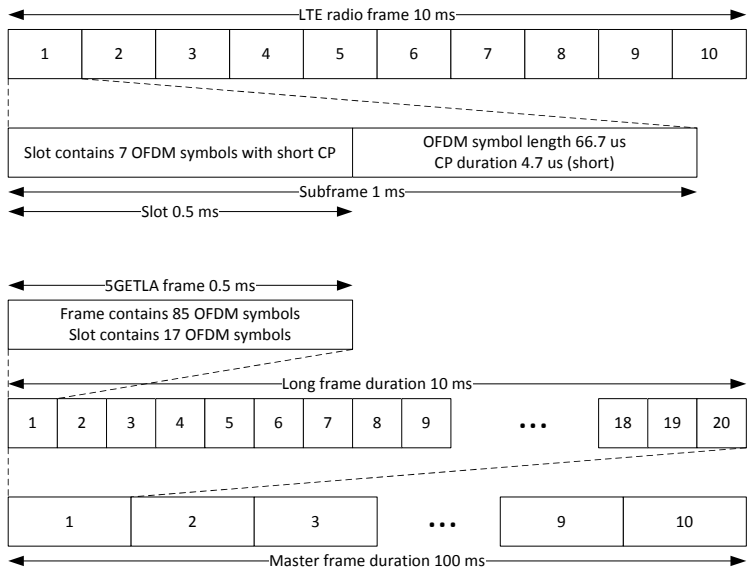

Fig. 4. Frame hierarchy for the 5GETLA system.

number of frames participating in the transmission.

\section{Higher Level Frame Hierarchy}

In Fig. 4, the higher level frame hierarchy for the 5GETLA is compared with the basic LTE frame design. Because one 5GETLA frame corresponds to a LTE slot duration, we chose the next logical element called Long Frame to correspond to the LTE radio frame, thus having length equal to $10 \mathrm{~ms}$. The largest logical element called Master Frame is currently chosen to be $100 \mathrm{~ms}$ long, based on typical beacon interval from WLAN networks. The $100 \mathrm{~ms}$ value is a compromise between the average delay caused by the long sleep cycle and the energy saving potentiality in the network and UE side [4]. In the case of very long operation time requirements for wireless nodes it should be possible for the network side to order even longer sleep periods for wireless sensors. With the frame structures presented in Fig. 4, we can support coexistence with overlay LTE macro cells and WLAN basic service sets (BSS). In the case of LTE overlay network, the eNB can schedule empty UL subframes in which the 5GBS would transceive information with nearby UE. Co-existence with a WLAN BSS can be achieved if the 5GBS and WLAN AP are able to agree on the share of the time resources. For example, co-existence with a WLAN AP can be achieved by reserving part of the WLAN AP transmit opportunity (TXOP) [22] time window for 5GBS.

In 5GETLA, the co-operation is noted by transmitting the chosen sleeping pattern in a 5GETLA master information block (MIB) once per Master Frame. The 5GETLA MIB is similar to LTE MIB, used to broadcast slowly changing system information once per master frame. The 5GETLA MIB provides minimum set of information elements that can be used by UE to select 5GBS to which it would be best to be associated. Note that in our design the UE may try to blindly associate with any 5GBS if desired. All the information to fully utilize the 5GETLA access, e.g., number of antennas in the 5GBS and number of supported spatial streams are then exchanged during the association process. 


\section{Benefits and Potential of the New Designs}

In this section we summarize the main benefits of the new designs and how they allow us to provide superior SE and EE performance compared to LTE/LTE-Advanced and IEEE 802.11 ac with reduced latency.

\section{A. Improved Spectral Efficiency}

Thanks to the very short frame duration and slowly changing channel conditions in a low mobility SCN, it is sufficient to estimate the UL and DL channel only once per frame or once per multiple frames. This significantly reduces the overhead of the CRS, DMRS, and CSIRS when compared to LTE-A, as indicated in [16]. In addition, by reducing the RB granularity in the frequency domain and simplifying the MAC layer we can achieve reduced control overhead. Typically, RSs and control fields are the dominant sources of overhead in system design. For example, the 5GETLA design achieves total overhead incorporating guard bands and CP to be $21.4 \%$ for SISO communications [4] and $26.4 \%$ for $8 \times 8$ DL SUMIMO scheme [16]. The corresponding overheads for LTE-A are $27.8 \%$ [4] and $37.4 \%$ [16], respectively.

\section{B. Reduced Latency}

By significantly reducing the OFDM symbol durations, frame durations, and by designing the DL and UL ACK responses within frame duration in the 5GETLA design, the PHY latency can be reduced to approximately one frame duration $T_{\text {frame }}=0.5 \mathrm{~ms}$. Furthermore, if we sacrifice part of the spectral efficiency and utilize FFT of size 256, we can further reduce the latency by half and achieve latency from resynchronization to DLCRS to first ACK to be less than $1 \mathrm{~ms}$, as indicated in [23]. With these values we are achieving the targets set in [1], [2], at least in the physical layer. In the $\mathrm{B} 4 \mathrm{G}$ design, the $T_{\text {frame }}=0.25 \mathrm{~ms}$ frame duration and the scheduling resource allocations of the next frame alleviate the buffering requirements when compared to 5GETLA design, and also lead to PHY RTT in the order of $1 \mathrm{~ms}$.

\section{Enhanced Energy Efficiency}

With reduced OFDM symbol durations, in addition to significantly improved latencies, we reduce the duty cycles of active devices enabling high efficiency sleep modes even in short sleep cycles, in which devices only listen to DLCRS and DLCCH or Tx Control in the case of 5GETLA or B4G design, respectively, and then return to sleep mode. When listening only to DLCRS and DLCCH, the devices are active (assuming ideal hardware) only 2 symbols out of 85 , leading to maximum sleep fraction of $97.6 \%$ of the time while maintaining capability to react to any transmitted frame. In the $\mathrm{B} 4 \mathrm{G}$ design, the maximum sleep fraction is approximately $92.9 \%$. In other words, significant power savings in the active UE are possible. If longer sleep cycles based on Long Frame and Master Frame time scales are used, the fraction of time slept increases even further.

In [8], an approximation on the achievable battery life between B4G numerology and LTE was shown, when assuming a constant time interval between receiving a short paging message and transmission only once per $1000 \mathrm{~s}$. In the case that the paging interval was $10 \mathrm{~ms}$, the LTE devices battery lifetime was only three days where as the B4G device could last for four months. When the paging interval was increased to $10 \mathrm{~s}$, the LTE device was able to operate for eight years, where as the B4G device was capable of achieving 55 year operation time. These results indicate how reducing the PHY latency does not only improve the system throughput, but it is also a key enabler for improved energy saving capabilities. Similar improvements can be expected from the 5GETLA design due to the reduced duty cycles.

In addition to improving mobile device battery durations as indicated in [8], the same applies to the network side and these new designs provide improved possibilities for short and long sleep cycles in the 5GBS. In the case of 5GETLA, if the 5GBS detects no activity inside certain time window it can turn to long sleep mode where it transmits only 5GETLA MIB packet and synchronization channel once per master frame. This maps to one active frame over 200 frames, leading to energy saving potential of $99.5 \%$ on the network side. Such a network sleep feature does not exist in current $3 \mathrm{GPP}$ solutions. If a $\mathrm{RACH}$ sequence or $\mathrm{CBDCH}$ packet is detected in the same frame, the 5GBS should be able to fully wake up during a few OFDM symbols in order to start operating in active mode in the next frame.

\section{Conclusions}

In this article, we have overviewed the 5GETLA and B4G radio interface designs which rely on OFDMA and agile, perframe reconfigurable TDD based scheduled and synchronized wireless access and compared them with each other. In these novel designs, the physical layer numerology is optimized for small cells taking into consideration the channel environment in small-cell communications. In addition, significant design effort to minimize the overhead of control fields and RS is given in both designs to provide superior spectral efficiency. Through minimized OFDM symbol durations, clearly reduced duty cycles are achieved when compared to LTE, which allows us to achieve clear improvements in energy efficiency and latency. Both designs achieve the $1 \mathrm{~ms}$ PHY latency target set for $5 \mathrm{G}$ wireless communications.

There remains several open challenges and research topics related to ultra-low latency radio interface design for dense $\mathrm{SCN}$. The most important open topics are listed below:

- modeling of the SINR statistics and inter-cell DL-UL collision statistics in very fast $\left(T_{\text {frame }} \leq 0.5 \mathrm{~ms}\right)$ flexible TDD based networks

- providing distributed mechanisms to allow 5G cells to adapt their DL-UL allocations while taking neighboring cells into consideration

- defining efficient scheduling algorithms for very fast TDD based radio interface with per-frame UL-DL reconfiguration

- designing spectrally efficient schemes to support sporadic small packet traffic

- fine-tuning the physical layer design to support enhanced interference cancellation schemes 
- supporting high mobility users through massive beamforming and possibly a modified RS layout design

This article is also seeking to catalyze the discussion on the possibilities of OFDMA and fast flexible TDD based beyond LTE-Advanced wireless access schemes, in addition to providing an overview of radio interface candidates for low latency, dense small-cell networks.

\section{ACKNOWLEDGMENT}

This work was partially supported by the Finnish Funding Agency for Technology and Innovation (Tekes), Broadcom Communications Finland and Huawei Finland, under the project "Energy-Efficient Wireless Networks and Connectivity of Devices - Densification (EWINE-D)".

\section{REFERENCES}

[1] Nokia Siemens Networks, "2020: Beyond 4G: Radio Evolution for the Gigabit Experience," [Online, last accessed $5^{\text {th }}$ September 2014], Available: http://nsn.com/sites/default/files/document/nokia_siemens_ networks_beyond_4g_white_paper_online_20082011_0.pdf, 2011.

[2] G. Fettweis and S. Alamouti, "5G: Personal mobile internet beyond what cellular did to telephony," Communications Magazine, IEEE, vol. 52, no. 2, pp. 140-145, February 2014.

[3] A. Frotzscher, U. Wetzker, M. Bauer, M. Rentschler, M. Beyer, S. Elspass, and H. Klessig, "Requirements and current solutions of wireless communication in industrial automation," in Communications Workshops (ICC), 2014 IEEE International Conference on, June 2014, pp. $67-72$.

[4] T. Levanen, J. Pirskanen, T. Koskela, J. Talvitie, and M. Valkama, "Radio Interface Evolution towards 5G and Enhanced Local Area Communications," Access, IEEE, vol. PP, no. 99, pp. 1-1, 2014.

[5] P. Mogensen, K. Pajukoski, B. Raaf, E. Tiirola, E. Lähetkangas, I. Kovacs, G. Berardinelli, L. Garcia, L. Hu, and A. Cattoni, "B4G local area: High level requirements and system design," in Globecom Workshops (GC Wkshps), 2012 IEEE, Dec 2012, pp. 613-617.

[6] P. Mogensen, K. Pajukoski, E. Tiirola, E. Lähetkangas, J. Vihriälä, S. Vesterinen, M. Laitila, G. Berardinelli, G. W. Da Costa, L. G. Garcia, F. M. Tavares, and A. F. Cattoni, "5G small cell optimized radio design," in Globecom Workshops (GC Wkshps), 2013 IEEE, Dec 2013, pp. 111116.

[7] E. Lahetkangas, K. Pajukoski, E. Tiirola, G. Berardinelli, I. Harjula, and J. Vihriala, "On the TDD subframe structure for beyond $4 \mathrm{G}$ radio access network," in Future Network and Mobile Summit (FutureNetworkSummit), 2013, July 2013, pp. 1-10.

[8] E. Lähetkangas, K. Pajukoski, J. Vihriälä, G. Berardinelli, M. Lauridsen, E. Tiirola, and P. Mogensen, "Achieving low latency and energy consumption by 5G TDD mode optimization," in Communications Workshops (ICC), 2014 IEEE International Conference on, June 2014, pp. 1-6.

[9] E. Dahlman, S. Parkvall, and J. Skold, 4G: LTE/LTE-Advanced for Mobile Broadband, 1st ed. Academic Press, 2011.

[10] M. Rinne and O. Tirkkonen, "LTE, the radio technology path towards 4G," Computer Communications, vol. 33, no. 16, pp. 1894 - 1906, 2010, [Online, last accessed $5^{\text {th }}$ September 2014], Available: http: //www.sciencedirect.com/science/article/pii/S0140366410003099.

[11] E. Perahia and R. Stacey, Next Generation Wireless LANs: 802.11n and $802.11 a c$, 2nd ed. New York, NY, USA: Cambridge University Press, 2013.
[12] "IEEE Standard for Information technology-Telecommunications and information exchange between systems-Local and metropolitan area networks-Specific requirements-Part 11: Wireless LAN Medium Access Control (MAC) and Physical Layer (PHY) Specifications-Amendment 4: Enhancements for Very High Throughput for Operation in Bands below 6 GHz." IEEE Std 802.11ac-2013 (Amendment to IEEE Std 802.112012, as amended by IEEE Std 802.11ae-2012, IEEE Std 802.11aa2012, and IEEE Std 802.11ad-2012), pp. 1-425, Dec 2013.

[13] "METIS Deliverable D2.2 - Novel radio link concepts and state of the art analysis," [Online, last accessed 30 $0^{\text {th }}$ October 2014], Available: https://www.metis2020.com/wp-content/uploads/deliverables/ METIS_D2.2_v1.pdf, Oct. 2013.

[14] A. A. Dowhuszko, O. Tirkkonen, J. Karjalainen, T. Henttonen, and J. Pirskanen, "A Decentraliced Cooperative Uplink/Downlink Adaptation Scheme for TDD Small Cell Networks," in Personal, Indoor and Mobile Radio Communications, 24th Annual IEEE International Symposium on, London, UK, 8-11 September 2013.

[15] G. Wunder, P. Jung, M. Kasparick, T. Wild, F. Schaich, Y. Chen, S. Brink, I. Gaspar, N. Michailow, A. Festag, L. Mendes, N. Cassiau, D. Ktenas, M. Dryjanski, S. Pietrzyk, B. Eged, P. Vago, and F. Wiedmann, "5GNOW: non-orthogonal, asynchronous waveforms for future mobile applications," Communications Magazine, IEEE, vol. 52, no. 2, pp. 97-105, February 2014.

[16] T. Levanen, J. Talvitie, J. Pirskanen, and M. Valkama, "New Spectrally and Energy Efficient Flexible TDD Based Air Interface for 5G Small Cells," in 2014 IEEE 79th Vehicular Technology Conference (VTC2014Spring) - The 2nd International Workshop on 5G Mobile and Wireless Communication System for 2020 and Beyond, Seoul, Korea, 18-21 May 2014, pp. 7-13.

[17] "3rd Generation Partnership Project; Technical Specification Group Radio Access Network; Evolved Universal Terrestrial Radio Access (EUTRA); User Equipment (UE) radio transmission and reception (Release 12)," 3GPP TS 36.101 V12.1.0 (2013-09), pp. 1-470, 2013.

[18] Z. Pi and F. Khan, "An introduction to millimeter-wave mobile broadband systems," Communications Magazine, IEEE, vol. 49, no. 6, pp. 101-107, June 2011.

[19] T. Levanen, J. Pirskanen, and M. Valkama, "Radio Interface Design for Ultra-Low Latency Millimeter-Wave Communications in 5G Era," in IEEE Global Communication Conference, Exhibition \& Industry Forum 2014 (IEEE Globecom 2014) - International Workshop on Ultra-Low Latency and Ultra-High Reliability in Wireless Communications, Austin, Texas, USA, 8-12 December 2014.

[20] Z. Shen, A. Khoryaev, E. Eriksson, and X. Pan, "Dynamic uplinkdownlink configuration and interference management in TD-LTE," Communications Magazine, IEEE, vol. 50, no. 11, pp. 51-59, 2012.

[21] R. Palit, K. Naik, and A. Singh, "Anatomy of WiFi Access Traffic of Smartphones and Implications for Energy Saving Techniques," International Journal of Energy, Information and Communications, vol. 3, no. 1, pp. 1-16, 2012, [Online, last accessed $5^{\text {th }}$ September 2014] Available: http://www.sersc.org/journals/IJEIC/vol3_Is1/1.pdf.

[22] "IEEE Standard for Information technology-Telecommunications and information exchange between systems-Local and metropolitan area networks-Specific requirements Part 11: Wireless LAN Medium Access Control (MAC) and Physical Layer (PHY) Specifications," IEEE Std 802.11-2012 (Revision of IEEE Std 802.11-2007), pp. 1-2793, 2012.

[23] T. Levanen, J. Pirskanen, T. Koskela, J. Talvitie, and M. Valkama, "Low latency radio interface for 5G flexible TDD local area communications," in Communications Workshops (ICC), 2014 IEEE International Conference on, June 2014, pp. 7-13. 